\title{
Adaptive Mutations Alter Antibody Structure and Dynamics during Affinity Maturation
}

\author{
Ramkrishna Adhikary, ${ }^{\dagger}$ Wayne Yu, ${ }^{\dagger}$ Masayuki Oda, ${ }^{\S}$ Ross C. Walker, $"$ Tingjian Chen,${ }^{\dagger}$ \\ Robyn L. Stanfield, ${ }^{\ddagger}$ Ian A. Wilson, ${ }^{\ddagger}$ Jörg Zimmermann, ${ }^{\dagger}$ and Floyd E. Romesberg ${ }^{* \dagger}$
}

\begin{abstract}
${ }^{\dagger}$ Department of Chemistry and ${ }^{\ddagger}$ Department of Integrative Structural and Computational Biology and The Skaggs Institute for Chemical Biology, The Scripps Research Institute, 10550 North Torrey Pines Road, La Jolla, California 92037, United States

${ }^{\S}$ Graduate School of Life and Environmental Sciences, Kyoto Prefectural University, 1-5, Hangi-cho, Shimogamo, Sakyo-ku, Kyoto 606-8522, Japan

"Department of Chemistry and Biochemistry and San Diego Supercomputer Center, University of California, San Diego, La Jolla, California 92093, United States
\end{abstract}

Supporting Information

\begin{abstract}
While adaptive mutations can bestow new functions on proteins via the introduction or optimization of reactive centers, or other structural changes, a role for the optimization of protein dynamics also seems likely but has been more difficult to evaluate. Antibody $(\mathrm{Ab})$ affinity maturation is an example of adaptive evolution wherein the adaptive mutations may be identified and Abs may be raised to specific targets that facilitate the characterization of protein dynamics. Here, we report the characterization of three affinity matured Abs that evolved from a common germline precursor to bind the chromophoric antigen (Ag), 8-methoxypyrene-1,3,6-trisulfonate
\end{abstract}

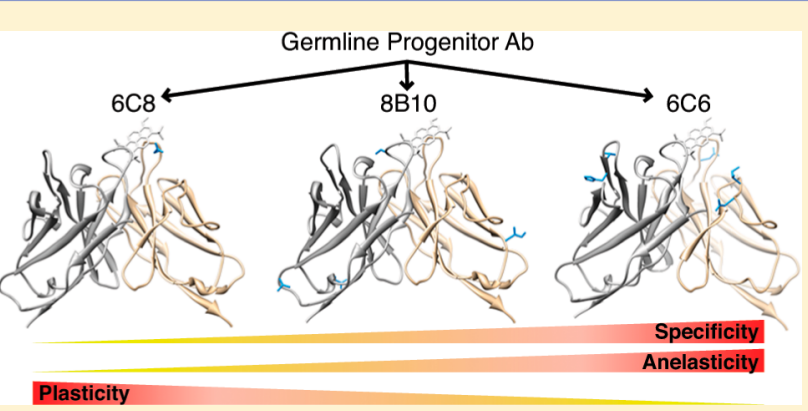
(MPTS). In addition to characterizing the sequence, molecular recognition, and structure of each Ab, we characterized the dynamics of each complex by determining their mechanical response to an applied force via three-pulse photon echo peak shift (3PEPS) spectroscopy and deconvoluting the response into elastic, anelastic, and plastic components. We find that for one Ab, affinity maturation was accomplished via the introduction of a single functional group that mediates a direct contact with MPTS and results in a complex with little anelasticity or plasticity. In the other two cases, more mutations were introduced but none directly contact MPTS, and while their effects on structure are subtle, their effects on anelasticity and plasticity are significant, with the level of plasticity correlated with specificity, suggesting that the optimization of protein dynamics may have contributed to affinity maturation. A similar optimization of structure and dynamics may contribute to the evolution of other proteins.

$\mathrm{T}$ he evolution of novel protein function is a hallmark of all biological systems and a subject of intense interest. A challenge to characterizing the process is that it is typically difficult, if not impossible, to unambiguously determine the specific adaptive mutations that conferred a new function because of complex genetic interactions and the presence of the many neutral mutations that accumulate on the time scale of evolution. In addition, while it is obvious that mutations may confer new activities by installing or optimizing functionality, or by introducing other changes to the protein's structure, ${ }^{1-3}$ dynamics may also be important. Indeed, it is dynamics that differentiates the limiting models of molecular recognition: flexibility is required for induced fit- or conformational selection-like recognition, ${ }^{4-7}$ and rigidity is required for lockand-key-like recognition. ${ }^{8}$ In addition, specificity is also an important selection pressure, and different levels of dynamics are inherently associated with different levels of specificity (just as flexibility allows for the adoption of structures involved in induced fit or conformational selection-like recognition, it will allow for the adoption of other conformations that recognize other targets). However, the characterization of protein dynamics is less straightforward than the characterization of structure, and the problem is further complicated by the fact that proteins have a vast number of internal motions, of which only a small subset is expected to contribute to a given function or to be subject to optimization during evolution.

Perhaps one of the most intuitive approaches to understanding the dynamics of any material is based on the response to an applied force. ${ }^{9-12}$ The resulting deformations may be characterized on the basis of the time scale of their response (Figure 1A): elastic deformations recover on the time scale of bond vibrations and arise from motions within a single potential energy minimum (e.g., inertial side-chain motions); anelastic deformations recover over time and arise from transitions between conformational substates separated by

Received: November 14, 2014

Revised: February 26, 2015

Published: March 10, 2015 
A

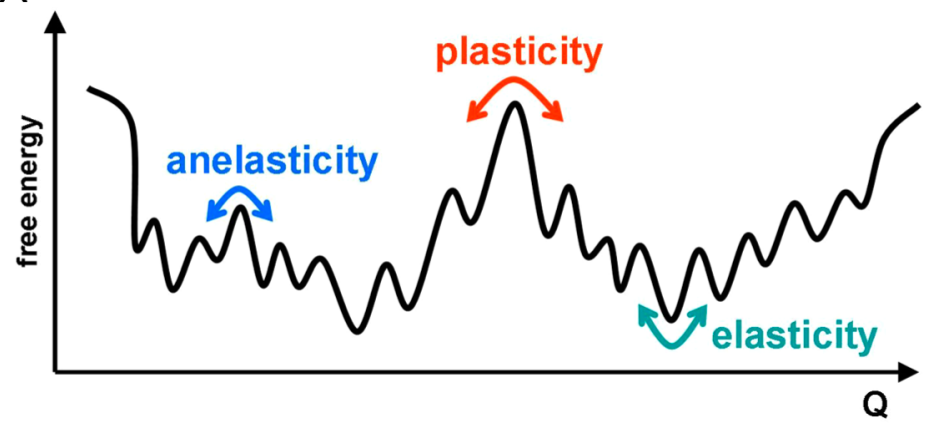

B

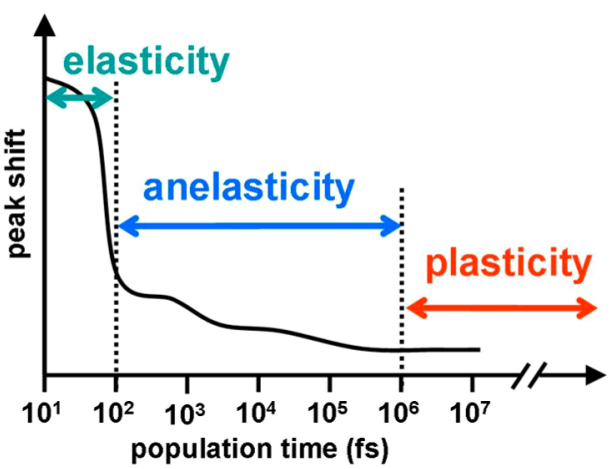

Figure 1. Schematic representation of barrier crossings on a protein free energy landscape (A) and peak shift decay (B) corresponding to elastic, anelastic, and plastic deformations. Coordinate $Q$ represents a projection of all internal degrees of freedom of the system.

relatively low barriers (e.g., ring flips and backbone fluctuations); and plastic deformations, although often defined as permanent because their time scale of recovery exceeds that of the experiment, recover on the longest time scale ${ }^{13,14}$ and correspond to transitions between states separated by high energy barriers (e.g., larger loop motions and conformational changes). To apply the same approach to the study of protein dynamics, a useful time scale for differentiating anelastic and plastic deformations is nanoseconds, the lifetime of an initial encounter complex. ${ }^{15,16}$ Although this time scale is significantly shorter than that typically used with bulk materials, it provides a functionally useful differentiation of protein dynamics as the time scales of elastic and anelastic deformations are then fast, allowing them to compete with dissociation of the encounter complex and thereby facilitate induced fit-like recognition, while plastic deformations are slow and produce the sufficiently long-lived conformational heterogeneity that defines conformational selection-like recognition. When combined with the requirement of lock-and-key mechanisms for relatively small deformations of any kind, this choice of time scale makes it possible to associate each mechanism of molecular recognition with a specific profile of dynamics.

In principle, modern ultrafast nonlinear optical methods, such as three-pulse photon echo peak shift (3PEPS) spectroscopy, ${ }^{17-19}$ are ideally suited to the characterization of protein dynamics because they reveal the response of a chromophore's environment to the force exerted by an excitation-dependent change in the chromophore's dipole moment. In a 3PEPS experiment, two laser pulses are used to inscribe a phase grating in the sample, which then decays due to environmental fluctuations caused by the relaxation of motions displaced by photoexcitation. This decay is monitored by applying a third pulse to the sample after a variable time delay that triggers rephasing of the chromophores whose environment remained unchanged and thereby results in stimulated emission (the echo signal). The decay of the stimulated emission, measured as a decay in the peak shift of its integrated intensity relative to time zero, ${ }^{17}$ reflects the time scales and relative amplitudes of environmental dynamics in the vicinity of the chromophore. Decays on the femto- to nanosecond time scale may be attributed to elasticity and anelasticity (depending on their precise time scale), while slower motions are manifest as a static offset in the 3PEPS decay, ${ }^{19,20}$ indicating the presence of plasticity (Figure 1B). Moreover, while the technique is sensitive only to motions that are coupled to the chromophore's transition dipole, if the chromophore can be associated specifically with the biologically relevant part of the protein (i.e., ligand or substrate binding site), then it enables the selective characterization of only those motions that are most likely to be biologically relevant.

One of the most remarkable examples of the evolution of molecular recognition is provided by the immune system, wherein antibodies (Abs) with specificity for virtually any foreign molecule [or antigen $(\mathrm{Ag})$ ] are evolved within days from a limited set of precursors (i.e., germline Abs). ${ }^{21}$ The Ag binds within a combining site that is formed from loops of hypervariable sequence (or complementarity-determining regions), three from the heavy-chain $\left(V_{H}\right)$ and three from the light-chain $\left(\mathrm{V}_{\mathrm{L}}\right)$ polypeptides $\left(\mathrm{V}_{\mathrm{H}}\right.$ CDR $1-3$ and $\mathrm{V}_{\mathrm{L}}$ CDR 1-3, respectively). A vast range of Ags may be recognized by a finite number of germline $\mathrm{Abs}$ because each $\mathrm{Ab}$ is polyspecific and able to recognize a broad range of targets with at least moderate affinity, thus triggering the introduction of adaptive mutations that optimize $\mathrm{Ag}$ recognition in a process known as affinity maturation. ${ }^{22-25} \mathrm{We}^{26-28}$ and others ${ }^{1,2}$ have suggested that the polyspecificity present in the repertoire of germline Abs results from high levels of flexibility and conformational heterogeneity and that the evolution of specificity during affinity maturation results, at least in part, from conformational restriction. We have also suggested that a similar modulation of protein dynamics may contribute to the evolution of altered specificity with other proteins. ${ }^{26-28}$ Regardless, by evolving Abs to a chromophoric Ag, the chromophore is by definition bound in a biologically relevant manner, and adaptive mutations may be unambiguously identified and their biologically relevant effects on molecular recognition characterized via 3PEPS spectroscopy.

To study the evolution of molecular recognition, we have applied 3PEPS spectroscopy to Abs that were evolved to bind the chromophoric Ag fluorescein ( $\mathrm{Fl}$ ) or 8-methoxypyrene1,3,6-trisulfonate [MPTS (Figure 2)]. We characterized panels<smiles>COc1cc([N+](=O)[O-])c2ccc3c([N+](=O)[O-])cc([S+](=O)[O-])c4ccc1c2c34</smiles>

Figure 2. Structure of MPTS. 
Table 1. X-ray Data Collection and Refinement Statistics ${ }^{a}$

\begin{tabular}{|c|c|c|}
\hline & 6C8-MPTS & 8B10-MPTS \\
\hline PDB entry & 4NJA & $4 \mathrm{NJ} 9$ \\
\hline beamline & APS 23-ID-D & APS 23-ID-D \\
\hline wavelength $(\AA)$ & 1.03318 & 1.03318 \\
\hline resolution $(\AA)$ & $42.60-2.20(2.24-2.20)$ & $32.90-1.95(1.98-1.95)$ \\
\hline space group & $P 2_{1} 2_{1} 2$ & $P 2_{1} 2_{1} 2_{1}$ \\
\hline cell dimensions $[a, b, c(\AA)]$ & $47.79,92.66,127.84$ & $37.13,58.44,212.85$ \\
\hline no. of observations & $118952(5656)$ & $198227(9671)$ \\
\hline no. of unique reflections & $28547(1414)$ & $35255(1727)$ \\
\hline completeness (\%) & $97.2(99.2)$ & $99.9(100.0)$ \\
\hline$R_{\text {sym }}(\%)$ & $7.5(55.5)$ & $7.0(66.8)$ \\
\hline$R_{\text {r.i.m. }}(\%)$ & $8.6(63.8)$ & $7.7(73.7)$ \\
\hline$R_{\text {p.i.m. }}(\%)$ & $3.7(27.2)$ & $3.2(31.1)$ \\
\hline$\langle I\rangle /\langle\sigma\rangle$ & $18.3(2.7)$ & $21.5(2.2)$ \\
\hline \multicolumn{3}{|c|}{ Refinement Statistics (all reflections $>0.0 \mathrm{~F} / \sigma \mathrm{F}$ ) } \\
\hline resolution $(\AA)$ & $42.60-2.20(2.26-2.20)$ & $32.90-1.95(2.20-1.95)$ \\
\hline no. of reflections (working set) & $27076(1742)$ & $33113(2167)$ \\
\hline no. of reflections (test set) & $1446(92)$ & $1752(105)$ \\
\hline$R_{\text {work }}(\%)$ & $20.7(34.2)$ & $21.4(33.6)$ \\
\hline$R_{\text {free }}(\%)$ & $25.7(38.8)$ & $26.3(37.0)$ \\
\hline no. of atoms (protein/MPTS/solvent) & $3338 / 30 / 151$ & $3286 / 30 / 254$ \\
\hline \multicolumn{3}{|l|}{ stereochemical parameters } \\
\hline Wilson $B\left(\AA^{2}\right)$ & 34.1 & 27.4 \\
\hline average isotropic $B\left(\AA^{2}\right)$, all atoms & 51.1 & 37.8 \\
\hline average isotropic $B\left(\AA^{2}\right)$, protein chains $(L, H)$ & $46.4,56.4$ & $36.9,38.5$ \\
\hline average isotropic $B\left(\AA^{2}\right)$, MPTS & 40.5 & 33.4 \\
\hline average isotropic $B\left(\AA^{2}\right)$, waters & 46.9 & 39.5 \\
\hline root-mean-square deviation for bond lengths $(\AA)$ & 0.015 & 0.017 \\
\hline root-mean-square deviation for bond angles ( $\mathrm{deg}$ ) & 1.82 & 1.87 \\
\hline \multirow[t]{3}{*}{ Ramachandran plot distribution from Molprobity } & $94.9 \%$ favored & $97.4 \%$ favored \\
\hline & $99.5 \%$ allowed & $100 \%$ allowed \\
\hline & $0.5 \%$ disallowed & $0.0 \%$ disallowed \\
\hline
\end{tabular}

${ }^{a} R_{\text {sym }}=\sum\left|I_{i}-\left\langle I_{i}\right\rangle / / \sum\right| I_{i} \mid$, where $I_{i}$ is the scaled intensity of the $i$ th measurement and $\left\langle I_{i}\right\rangle$ is the mean intensity for that reflection. Estimated $R_{\mathrm{r} . \mathrm{m} . \mathrm{m}}=$ $R_{\text {sym }}[N /(N-1)]^{1 / 2}$, where $N$ is data multiplicity. Estimated $R_{\text {p.i.m. }}=R_{\text {sym }}[1 /(N-1)]^{1 / 2}$, where $N$ is data multiplicity. $R_{\text {cryst }}=\sum\left\|F_{\text {obs }}|-| F_{\text {calc }}\right\| / \sum$ $F_{\text {obs }}$, where $F_{\text {calc }}$ and $F_{\text {obs }}$ are the calculated and observed structure factor amplitudes, respectively. $R_{\text {free }}$ as for $R_{\text {cryst }}$ but for $5.0 \%$ of the total reflections chosen at random and omitted from refinement.

of both anti-Fl and anti-MPTS Abs to examine the range of dynamics present within the different Abs. ${ }^{26,27,29,30}$ In the case of one anti-Fl $\mathrm{Ab}$, we characterized dynamics as a function of affinity maturation and found that it reduced both anelasticity and plasticity, suggesting that Abs are indeed conformationally restricted during affinity maturation. ${ }^{28}$ Here, we report the identification of three novel anti-MPTS Abs, 6C8, 8B10, and 6C6. Interestingly, the three Abs were found to have diverged from a common ancestral germline, and thus, any differences are by definition the result of the different mutations acquired. Characterization of the Abs revealed that while each evolved more optimal MPTS recognition, they did so via different mechanisms that are associated with rather different overall profiles of molecular recognition. Interestingly, for one $A b$, the unique profile of molecular recognition resulted from the introduction of a specific functional group, while for the other Abs, the structural changes are more subtle and the differences in molecular recognition appear to have resulted at least in part from relatively larger changes in anelasticity and/or plasticity. The results suggest that the optimization of both structure and dynamics likely contributes to Ab affinity maturation and, by extension, to the evolution of novel function with other proteins.

\section{MATERIALS AND METHODS}

Generation and Sequencing of Anti-MPTS Abs. Abs were raised in Swiss Webster mice via immunization with MPTS conjugated to keyhole limpet hemocyanin (KLH), and monoclonal IgG antibodies were produced and purified from hybridoma supernatants by standard methods. ${ }^{31}$ To sequence the heavy-chain variable $\left(\mathrm{V}_{\mathrm{H}}\right)$ and light-chain variable $\left(\mathrm{V}_{\mathrm{L}}\right)$ gene regions, mRNA was extracted from fresh hybridoma cells (Qiagen RNeasy Kit) and used with oligonucleotide $(\mathrm{dT})_{18}$ to synthesize cDNA (SuperScript III Kit, Invitrogen). The $\mathrm{V}_{\mathrm{H}}$ and $\mathrm{V}_{\mathrm{L}}$ genes from the resulting cDNA were amplified via polymerase chain reaction using a specifically designed primer set for cloning mouse immunoglobulin regions $s^{32,33}$ and then sequenced. Nucleotide sequences of the $V_{H}$ and $V_{L}$ genes are provided in the Supporting Information.

Isothermal Titration Calorimetry (ITC). Calorimetric titration of each $\mathrm{Ab}$ with MPTS in $1 \times$ PBS [phosphatebuffered saline ( $\mathrm{pH} 7.4)]$ was performed at 20,25 , and $30^{\circ} \mathrm{C}$. For each ITC experiment, a $6 \mu \mathrm{M}$ solution of antibody was titrated by injecting a $120 \mu \mathrm{M}$ solution of MPTS. Each titration consisted of a preliminary $0.6 \mu \mathrm{L}$ injection followed by 19 subsequent $2 \mu \mathrm{L}$ additions, which were performed over $4 \mathrm{~s}$ periods at $120 \mathrm{~s}$ intervals. Thermodynamic parameters of $\mathrm{Ag}$ 


$$
\begin{array}{llllllllll}
1 & 10 & 20 & \text { abcd } 30 & 40 & 50 & 60 & 70 & 80 & 90
\end{array}
$$

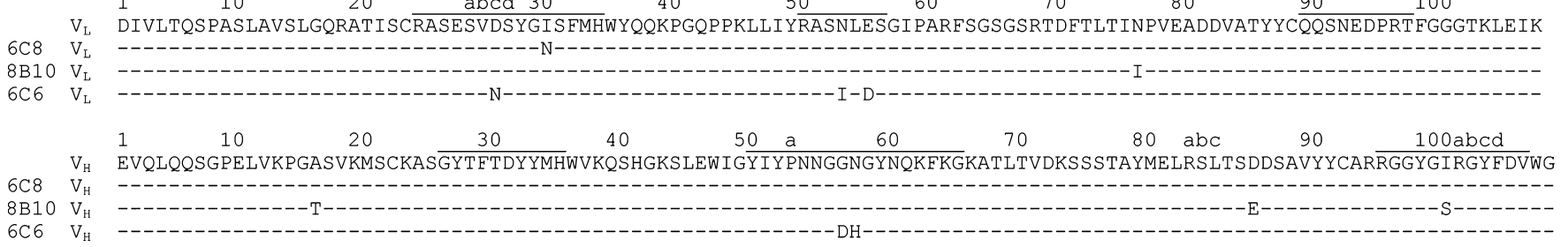

Figure 3. Amino acid sequences of the anti-MPTS Abs. Kabat numbering ${ }^{50}$ is shown at the top, with the CDRs underlined. The consensus sequence is listed first with identical residues in the three anti-MPTS Abs indicated with a dash.

binding are provided in Table 2 and the Supporting Information.

Enzyme-Linked Immunosorbent Assay (ELISA) Procedure. Each well of a 96-well plate was coated with one of 45 randomly selected proteins (see Table S3 of the Supporting Information) by addition of $100 \mu \mathrm{L}$ of a stock solution and incubation at $4{ }^{\circ} \mathrm{C}$ overnight. Next, the wells were each washed three times with $150 \mu \mathrm{L}$ of $1 \times \mathrm{PBS}$, and then blocked with 150 $\mu \mathrm{L}$ of $3 \%$ BSA in $1 \times$ PBS by incubation at $37^{\circ} \mathrm{C}$ for $2 \mathrm{~h}$. Next, $80 \mu \mathrm{L}$ of a stock solution of each primary antibody (11.5 $\mu \mathrm{g} /$ $\mathrm{mL}$ for $6 \mathrm{C} 8,9.0 \mu \mathrm{g} / \mathrm{mL}$ for $8 \mathrm{~B} 10$, and $12.3 \mu \mathrm{g} / \mathrm{mL}$ for $6 \mathrm{C} 6$ ) was added to each well, and the plates were incubated at $37^{\circ} \mathrm{C}$ for $2 \mathrm{~h}$. Wells were then washed six additional times with 200 $\mu \mathrm{L}$ of ELISA washing buffer ( $0.05 \%$ Tween 20 in $1 \times$ PBS). After the wash step, $100 \mu \mathrm{L}$ of secondary antibody (1:2000 diluted chicken anti-mouse $\operatorname{IgG} \mathrm{Fc}$ secondary $\mathrm{Ab}$ conjugated with HRP, Pierce/Thermo Scientific, Rockford, IL) was added to each well and incubated at $37^{\circ} \mathrm{C}$ for $2 \mathrm{~h}$. After six additional washes with ELISA washing buffer, $200 \mu \mathrm{L}$ of a freshly prepared $o$-phenylenediamine (OPD) (Sigma-Aldrich, St. Louis, MO)/ $\mathrm{H}_{2} \mathrm{O}_{2}$ solution was added to each well. After $20 \mathrm{~min}$, the reaction was quenched with $50 \mu \mathrm{L}$ of $3 \mathrm{M} \mathrm{H}_{2} \mathrm{SO}_{4}$, and absorbance at $492 \mathrm{~nm}$ was recorded (EnVision 2103 Multilabel Reader, PerkinElmer). For quantification, a control well for each of the 45 proteins was treated identically, with the exception that no primary $\mathrm{Ab}$ was added, and the absolute absorbance for the experimental well was calculated by subtraction. ELISA data for each protein and antibody were collected in triplicate.

X-ray Crystallography. Abs 8B10 (mouse, IgG2a, kappa) and $6 \mathrm{C} 8$ (mouse, IgG2b, kappa) IgG were purified from ascites fluid by standard methods and digested to $\mathrm{Fab}$ or $\mathrm{F}\left(\mathrm{ab}^{\prime}\right)_{2}$ with papain or pepsin, respectively. Papain (Sigma) was activated with $10 \mathrm{mM}$ cysteine and $3 \mathrm{mM}$ EDTA in $0.1 \mathrm{M}$ sodium acetate (pH 5.5) at $37{ }^{\circ} \mathrm{C}$ for $15 \mathrm{~min}$. Purified 8B10 IgG was digested with $2 \%(\mathrm{w} / \mathrm{w})$ activated papain for $2 \mathrm{~h}$ at $37^{\circ} \mathrm{C}$, and the reaction was stopped by addition of iodoacetamide to a final concentration of $30 \mathrm{mM}$. The $6 \mathrm{C} 8 \mathrm{IgG}$ solution was adjusted to $\mathrm{pH} 3.5$ with $1.0 \mathrm{M}$ sodium acetate and then digested with pepsin $[4 \%(\mathrm{w} / \mathrm{w})($ Sigma $)]$ for $3 \mathrm{~h}$ at $37^{\circ} \mathrm{C}$. The reaction was stopped by addition of $3 \mathrm{M}$ Tris to a final $\mathrm{pH}$ of $7 . \mathrm{F}\left(\mathrm{ab}^{\prime}\right)_{2}$ was then reduced to $\mathrm{Fab}^{\prime}$ with $15 \mathrm{mM}$ cysteine $(\mathrm{pH} 7)$ for $2 \mathrm{~h}$, and the reaction was stopped by adding iodoacetamide to a final concentration of $30 \mathrm{mM}$. In both cases, $\mathrm{Fab}$ or $\mathrm{Fab}^{\prime}$ was separated from $\mathrm{Fc}$ by affinity chromatography (HiTrap protein A, GE healthcare). Both Fabs were then further purified by size exclusion chromatography using a Superdex 200 16/60 column (GE Healthcare) in $20 \mathrm{mM}$ Tris and $150 \mathrm{mM} \mathrm{NaCl}$ (pH 7.5).

In the same buffer used for size exclusion chromatography, Fabs were concentrated to 9.2 and $4.8 \mathrm{mg} / \mathrm{mL}$ for $8 \mathrm{~B} 10$ and $6 \mathrm{C} 8$, respectively, and then crystallized as follows. An aqueous MPTS $(50 \mathrm{mg} / \mathrm{mL})$ solution was added to Fab in a 10:1
(MPTS:Fab) molar excess, and the Fab-MPTS complexes were screened for crystallization with the JCSG/IAVI/TSRI Crystalmation system (Rigaku) using the JCSG Core Suite (Qiagen) reagent kit followed by manual optimization of promising conditions. Crystals of the 6C8-MPTS complex were grown in sitting drops $(0.4 \mu \mathrm{L}$ of protein and $0.4 \mu \mathrm{L}$ of well solution) against $20 \mathrm{mM}$ zinc acetate, $12 \%$ PEG 8000, and $0.1 \mathrm{M}$ MES (pH 6.5) at $4{ }^{\circ} \mathrm{C}$. Crystals of the 8B10-MPTS complex were grown in sitting drops $(0.4 \mu \mathrm{L}$ of protein and 0.4 $\mu \mathrm{L}$ of well solution) against $200 \mathrm{mM}$ zinc acetate and $18 \%$ PEG 3350 at $20{ }^{\circ} \mathrm{C}$. Crystals were cryoprotected by brief immersion in a mixture of $30 \%$ glycerol and $70 \%$ well solution $(6 \mathrm{C} 8-$ MPTS) or 30\% ethylene glycol and 70\% well solution (8B10MPTS) and then flash-cooled in liquid nitrogen. For both complexes, data were collected from a single crystal at APS beamline 23-ID-D. The 8B10 structure was determined by molecular replacement using Phaser $^{34}$ and the coordinates of Fab 29G12 [Protein Data Bank (PDB) entry 1MEX] as a model, and the $6 \mathrm{C} 8$ structure was determined in a similar fashion using the $8 \mathrm{~B} 10$ coordinates as a model. In both cases, the Fab search models were split into separate variable and constant domains to allow for variation in the Fab elbow angle. Structures were refined with Refmac5, ${ }^{35}$ and model building was performed with Coot. ${ }^{36}$ Both structures had one FabMPTS complex in the asymmetric unit. Data collection statistics and final refinement statistics are listed in Table 1, and a superposition of the two complexes is shown in Figure S5 of the Supporting Information. Coordinates and data have been deposited in the Protein Data Bank as entries 4NJ9 for the 8B10-MPTS complex and 4NJA for the 6C8-MPTS complex.

3PEPS Spectroscopy. All samples were prepared in $1 \times$ PBS (pH 7.4) using a stock solution of $2.9 \mathrm{mg} / \mathrm{mL}$ for $6 \mathrm{C} 8,2.6$ $\mathrm{mg} / \mathrm{mL}$ for $8 \mathrm{~B} 10,3.9 \mathrm{mg} / \mathrm{mL}$ for $6 \mathrm{C} 6$, and $4.95 \mathrm{mM}$ for MPTS. The required amount of MPTS and $\mathrm{Ab}(\mathrm{IgG})$ stock solutions were mixed such that a concentration of $700 \mu \mathrm{M}$ of the MPTS $-\mathrm{Ab}$ complex was achieved when the total solution was concentrated to a final volume of $100 \mu \mathrm{L}$. To ensure complete removal of unbound MPTS from the Ab-MPTS complexes, samples were washed by repeated dilution with $1 \times$ PBS and microfiltration using a $10 \mathrm{kDa}$ molecular weight cutoff centrifugal filter unit (Amicon Ultra, Millipore) until the filtrate was free of MPTS as determined by UV-vis absorption. Absorption spectra of free and bound MPTS are shown in Figure S2 of the Supporting Information.

A detailed description of the experimental setup for 3PEPS has been reported previously. ${ }^{26}$ Briefly, the frequency-doubled pulses ( $\sim 45 \mathrm{fs}, 416 \mathrm{~nm}, 200 \mathrm{~nJ})$ of the fundamental beam ( 836 $\mathrm{nm}$ ) of a Ti:sapphire regenerative amplifier (Spitfire, Spectra Physics) was used as the excitation source. The excitation beam was split into three, roughly equal portions, arranged in an equilateral triangle, and focused using a plano-convex lens (200 $\mathrm{mm}$ focal length) on the sample contained in a spinning cell 
with a path length of $250 \mu \mathrm{m}$. Each excitation pulse was $\sim 20-$ $25 \mathrm{~nJ}$. The photon echo signals from the Ab-MPTS sample in the two phase-matched directions $k_{1}-k_{2}+k_{3}$ and $-k_{1}+k_{2}+k_{3}$ were detected with two large area avalanche photodiodes (Advanced Photonics). At least three independent experiments were performed for each $\mathrm{Ab}$. The 3PEPS decays were fit using a model spectral density function formalism ${ }^{20}$ (for details, see the Supporting Information). Data fitting was performed on the 64bit Linux cluster Garibaldi at The Scripps Research Institute using a custom suite of $\mathrm{C}$ programs developed in our laboratory based on the code provided by D. Larsen (University of California, Davis, CA). For representative 3PEPS fits and further experimental details, see the Supporting Information.

\section{RESULTS}

Characterization of $\mathrm{Ab}$ Evolution and Molecular Recognition. From a panel of anti-MPTS Abs, we identified three, $6 \mathrm{C} 8,8 \mathrm{~B} 10$, and $6 \mathrm{C} 6$, that are homologous and share virtually identical $\mathrm{V}_{\mathrm{H}}$ recombination junctions, ${ }^{22,37,38}$ indicating that they arose from a common germline precursor (Figure 3 ). Thus, the sequence differences may be ascribed to affinity maturation. Consistent with the conclusion, the consensus sequences of the light and heavy chains are virtually identical to the germline genes IGKV3-5*01 F and IGHV1-34*01 F, respectively. ${ }^{39}$ This analysis reveals that $\mathrm{Ab} 6 \mathrm{C} 8$ evolved via a single $\mathrm{V}_{\mathrm{L}}$ mutation, $\mathrm{I} 30 \mathrm{~N}\left(\mathrm{I}^{\mathrm{L}} 30 \mathrm{~N}\right)$; $\mathrm{Ab} 8 \mathrm{~B} 10$ evolved via four mutations, $\mathrm{N}^{\mathrm{L}} 76 \mathrm{I}, \mathrm{A}^{\mathrm{H}} 16 \mathrm{~T}, \mathrm{D}^{\mathrm{H}} 85 \mathrm{E}$, and $\mathrm{I}^{\mathrm{H}} 100 \mathrm{~S}$, and $\mathrm{Ab} 6 \mathrm{C} 6$ evolved via five mutations, $\mathrm{D}^{\mathrm{L}} 27 \mathrm{cN}, \mathrm{N}^{\mathrm{L}} 53 \mathrm{I}, \mathrm{E}^{\mathrm{L}} 55 \mathrm{D}, \mathrm{G}^{\mathrm{H}} 56 \mathrm{D}$, and $\mathrm{N}^{\mathrm{H}} 57 \mathrm{H}$ (Kabat numbering ${ }^{40}$ ).

To characterize the recognition of MPTS, isothermal titration calorimetry was used to determine the dissociation constant $\left(K_{\mathrm{D}}\right)$ and binding enthalpy $\left(\Delta H^{\circ}\right)$ for each sibling $\mathrm{Ab}$, and these values were used to calculate the binding free energy $\left(\Delta G^{\circ}\right)$ and entropy $\left(\Delta S^{\circ}\right)$ (Table 2). Ab 6C6 binds MPTS

Table 2. Thermodynamic Parameters at $298 \mathrm{~K}$

\begin{tabular}{lcccc} 
& $\begin{array}{c}K_{\mathrm{D}} \\
(\mathrm{nM})\end{array}$ & $\begin{array}{c}\Delta G^{\circ} \\
(\mathrm{kcal} / \mathrm{mol})\end{array}$ & $\begin{array}{c}\Delta H^{\circ} \\
(\mathrm{kcal} / \mathrm{mol})\end{array}$ & $\begin{array}{c}T \Delta S^{\circ} \\
(\mathrm{kcal} / \mathrm{mol})\end{array}$ \\
\hline $6 \mathrm{C} 8$ & $600 \pm 100$ & $-8.5 \pm 0.1$ & $-8.6 \pm 0.04$ & $-0.1 \pm 0.1$ \\
8B10 & $108 \pm 8$ & $-9.6 \pm 0.1$ & $-11.11 \pm 0.01$ & $-1.5 \pm 0.1$ \\
$6 \mathrm{C} 6$ & $36 \pm 7$ & $-10.2 \pm 0.1$ & $-10.96 \pm 0.03$ & $-0.7 \pm 0.1$ \\
\hline
\end{tabular}

with the highest affinity $(\sim 40 \mathrm{nM})$, resulting from a favorable $\Delta H^{\circ}$ as well as a relatively favorable $\Delta S^{\circ}$, while $\mathrm{Ab} 8 \mathrm{~B} 10$ binds MPTS with an intermediate affinity $(\sim 100 \mathrm{nM})$. Ab $6 \mathrm{C} 8$ binds MPTS with the lowest affinity $(\sim 600 \mathrm{nM})$ of the three siblings because of a less favorable $\Delta H^{\circ}$, despite a more favorable $\Delta S^{\circ}$.

To more broadly characterize the molecular recognition of each $\mathrm{Ab}$, we determined their relative affinities for 45 randomly selected proteins via a standard ELISA (Figure 4). The broadest range of proteins is recognized by $\mathrm{Ab} 6 \mathrm{C} 8$. Compared to $\mathrm{Ab}$ $6 \mathrm{C} 8, \mathrm{Ab} 6 \mathrm{C} 6$ binds eight proteins more tightly, 11 with similar affinity, and 26 with lower affinity. In contrast, scarcely any of the proteins are recognized by $\mathrm{Ab} 8 \mathrm{~B} 10$, which is thus apparently more specific for MPTS.

Characterization of $\mathrm{Ab}$ Structure. Crystal structures of the 8B10-MPTS and 6C8-MPTS complexes were determined by molecular replacement to 1.95 and $2.20 \AA$ resolution, respectively, with one Fab-MPTS complex per asymmetric unit. The two complexes are similar with a root-mean-square deviation of only $0.42 \AA$ for all $\mathrm{C}_{\alpha}$ atoms within the variable domains (Figure 5). In each case, MPTS is bound in a similar
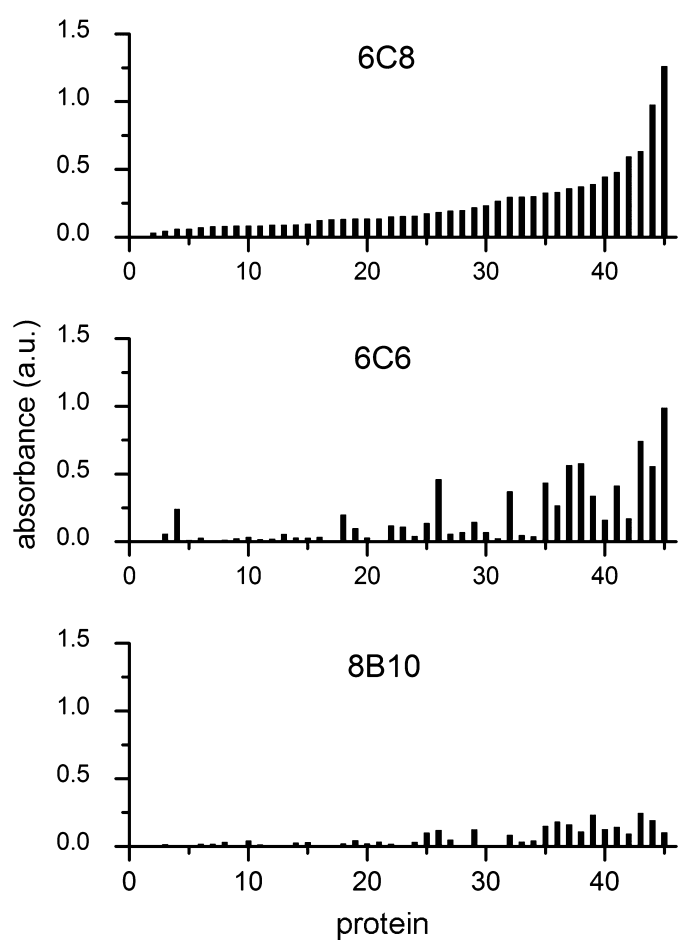

Figure 4. Repertoire of proteins recognized by each anti-MPTS Ab as determined by an ELISA. The 45 randomly selected proteins (see Table S3 of the Supporting Information) are arranged according to the affinity with which they are bound by $6 \mathrm{C} 8$. Data were collected in triplicate, and bars represent the average absorbance normalized by $\mathrm{Ab}$ concentration.

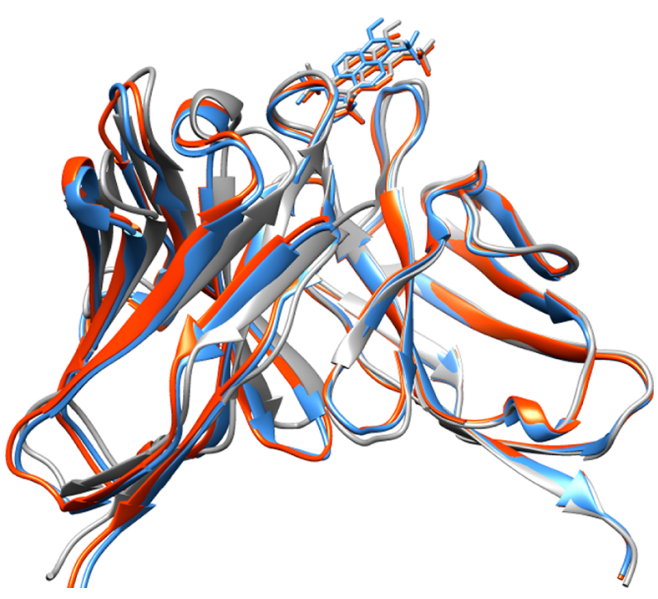

Figure 5. Overlay of the $6 \mathrm{C} 8$ (blue) and $8 \mathrm{~B} 10$ (red) crystal structures, and the 6C6 (gray) model structure based on the superposition of the constant regions.

fashion in a shallow pocket and buries $\sim 230$ and $\sim 250 \AA^{2}$ of MPTS and Ab surface area, respectively (MS, $1.7 \AA$ probe radius $),{ }^{41}$ with $\sim 55 \%$ of the buried $\mathrm{Ab}$ surface provided by the $\mathrm{V}_{\mathrm{L}}$. The binding site is located on one face of $\mathrm{V}_{\mathrm{H}} \mathrm{CDR} 3$ and is formed by $\mathrm{Gly}^{\mathrm{H}} 97, \mathrm{Tyr}^{\mathrm{H}} 98, \mathrm{Gly}^{\mathrm{H}} 99, \operatorname{Ser}^{\mathrm{H}} 100, \operatorname{Arg}^{\mathrm{H}} 100 \mathrm{a}$, Gly ${ }^{\mathrm{H}} 100 \mathrm{~b}$, and $\mathrm{Tyr}^{\mathrm{H}} 100 \mathrm{c}$, as well as $\mathrm{Tyr}^{\mathrm{L}} 28, \mathrm{Il}^{\mathrm{L}} 30$, $\mathrm{Phe}^{\mathrm{L}} 32$, $\operatorname{Arg}^{\mathrm{L}} 50$, and $\mathrm{Asn}^{\mathrm{L}} 53$ of $\mathrm{V}_{\mathrm{L}}$ CDR1 and CDR2. Ile ${ }^{\mathrm{L}} 30$ and $\mathrm{Phe}^{\mathrm{L}} 32$ form the floor of the binding pocket, while $\mathrm{V}_{\mathrm{H}} \mathrm{CDR} 3, \operatorname{Arg}^{\mathrm{L}} 50$, and $\mathrm{Tyr}^{\mathrm{L}} 28$ form a wall of the pocket that separates MPTS from the geometrical center of the $\mathrm{Ab}$ combining site. Two of the three MPTS sulfonate groups are oriented toward this wall, where a significant level of specificity appears to be mediated by 
Table 3. Fit Parameters for 3PEPS Data

\begin{tabular}{|c|c|c|c|c|c|c|c|c|}
\hline & \multicolumn{3}{|c|}{ elastic } & \multicolumn{4}{|c|}{ anelastic } & \multirow{2}{*}{$\frac{\text { plastic }}{\Delta_{\text {inh }}\left(\mathrm{cm}^{-1}\right)}$} \\
\hline & $\lambda_{\mathrm{BO}}\left(\mathrm{cm}^{-1}\right)$ & $\omega_{\mathrm{BO}}\left(\mathrm{cm}^{-1}\right)^{a}$ & $\Gamma_{\mathrm{BO}}\left(\mathrm{cm}^{-1}\right)^{a}$ & $\lambda_{\mathrm{K} 1}\left(\mathrm{~cm}^{-1}\right)$ & $\overline{\tau_{\mathrm{K} 1}(\mathrm{fs})}$ & $\lambda_{\mathrm{K} 2}\left(\mathrm{~cm}^{-1}\right)$ & $\tau_{\mathrm{K} 2}(\mathrm{ps})$ & \\
\hline $6 \mathrm{C} 8$ & $430 \pm 30$ & 550 & 320 & $103 \pm 8$ & $500 \pm 160$ & $58 \pm 14$ & $9.8 \pm 2.6$ & $2 \pm 1$ \\
\hline $8 \mathrm{~B} 10$ & $414 \pm 20$ & 550 & 320 & $98 \pm 7$ & $470 \pm 110$ & $98 \pm 18$ & $4.7 \pm 1.2$ & $0 \pm 1$ \\
\hline $6 \mathrm{C} 6$ & $420 \pm 30$ & 550 & 320 & $65 \pm 36$ & $580 \pm 120$ & $97 \pm 7$ & $4.7 \pm 2.3$ & $138 \pm 10$ \\
\hline
\end{tabular}

five H-bonds, which are the only intermolecular $\mathrm{H}$-bonds formed in the $8 \mathrm{~B} 10$ and $6 \mathrm{C} 8$ complexes. Specifically, one sulfonate group forms an $\mathrm{H}$-bond with the backbone $\mathrm{NH}$ moieties of $\mathrm{Tyr}^{\mathrm{H}} 98$ and $\mathrm{Gly}^{\mathrm{H}} 99$, and the side chain of $\operatorname{Arg}^{\mathrm{L}} 50$, while the second sulfonate group forms $\mathrm{H}$-bonds with both the $\mathrm{NH}$ backbone and side chain of $\operatorname{Arg}^{\mathrm{H}} 100$. In the $8 \mathrm{~B} 10$ complex, the methoxy group and remaining sulfonate group on the opposite side of MPTS are not engaged by the Ab. The $\mathrm{I}^{\mathrm{L}} 30 \mathrm{~N}$ somatic mutation in $6 \mathrm{C} 8$ is located in the floor of the pocket and introduces a side-chain $\mathrm{H}$-bond with the third sulfonate group of MPTS, which results in the Ag being simultaneously engaged from opposite sides. A model for the 6C6-MPTS complex was constructed using the $6 \mathrm{C} 8-\mathrm{MPTS}$ complex as a template (for details, see the Supporting Information). Comparison of the 6C6 model with the crystal structures revealed a similar binding pocket with the same five $\mathrm{H}$-bonds observed in the $8 \mathrm{~B} 10$ structure. As expected, the framework regions of all three complexes were superimposable, the largest differences being observed in the CDR loops (Figure 5). Nonetheless, the differences were still small among the CDR loops that form the MPTS binding site $\left(V_{L}\right.$ CDR1, $V_{L}$ CDR2, $\mathrm{V}_{\mathrm{L}} \mathrm{CDR} 3$, and $\mathrm{V}_{\mathrm{H}} \mathrm{CDR} 3$ ), and larger differences were observed only with the more distal $\mathrm{V}_{\mathrm{H}}$ CDR1 and $\mathrm{V}_{\mathrm{H}}$ CDR2 loops.

Characterization of $\mathbf{A b}$ Dynamics. 3PEPS characterization of each $\mathrm{Ab}-\mathrm{MPTS}$ complex revealed four time scales of dynamics (Table 3 and Figure 6). Each complex showed a similar sub-100 fs decay that is attributed to elastic deformations associated with water molecules or charged protein side chains. To fit this decay component, the frequency $\left(\omega_{\mathrm{BO}}\right)$ and damping constant $\left(\Gamma_{\mathrm{BO}}\right)$ of a Brownian oscillator function were fixed to $550 \mathrm{fs}$ and $320 \mathrm{~cm}^{-1}$, respectively, and

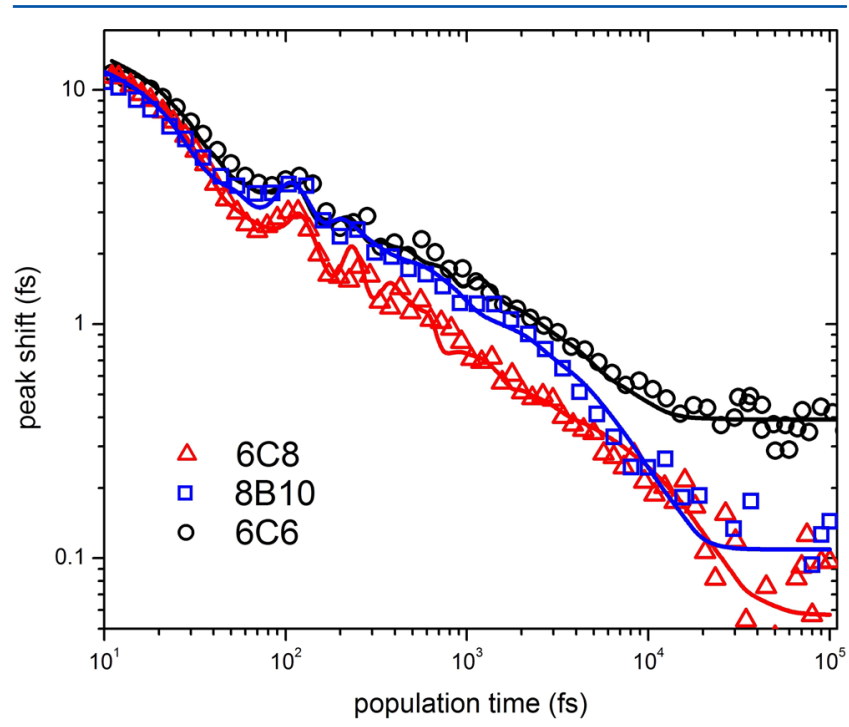

Figure 6. 3PEPS decays for Abs 6C8, 8B10, and 6C6 (symbols, data points; lines, best fit to data). the reorganization energy $\left(\lambda_{\mathrm{BO}}\right)$ was allowed to vary freely, yielding a value of $\sim 400 \mathrm{~cm}^{-1}$ for each Ab-MPTS complex. Each complex also exhibited two picosecond time scale motions associated with anelastic deformations that were fit with a Kubo function. The faster of these decays is again similar among all the complexes, with time constants $\left(\tau_{\mathrm{K} 1}\right)$ of $\sim 0.5 \mathrm{ps}$ and reorganization energies $\left(\lambda_{\mathrm{K} 1}\right)$ of $\sim 90 \mathrm{~cm}^{-1}$, and is attributed to protein motions or to the motion of proximal water molecules, which are known to cause echo dephasing on this time scale. ${ }^{26}$ The slower picosecond decay is similar in $8 \mathrm{~B} 10$ and $6 \mathrm{C} 6\left(\tau_{\mathrm{K} 2}=\right.$ $4.7 \mathrm{ps}$, and $\left.\lambda_{\mathrm{K} 2} \sim 100 \mathrm{~cm}^{-1}\right)$, but has a longer time scale $\left(\tau_{\mathrm{K} 2}=\right.$ $9.8 \mathrm{ps})$ and a lower amplitude $\left(\lambda_{\mathrm{K} 2}=58 \mathrm{~cm}^{-1}\right)$ with $6 \mathrm{C} 8$. Decays on this time scale are not observed for MPTS in solution and are thus attributed to protein motions. ${ }^{26}$ Finally, $\mathrm{Ab} 6 \mathrm{C} 6$ alone showed a long time signal offset $\left(\Delta_{\text {inh }}=138\right.$ $\mathrm{cm}^{-1}$ ), which corresponds to a decay on time scales longer than the experimental time window $(\sim 1 \mathrm{~ns})$ and is assigned to protein motions that interconvert different conformations of the protein, and thus to protein plasticity. ${ }^{28,29}$

\section{DISCUSSION}

The hallmark of adaptive immunity is the evolution of Abs with exquisite specificity for virtually any foreign $\mathrm{Ag}$ from a relatively small number of precursor germline Abs. Interestingly, Abs $6 \mathrm{C} 8,8 \mathrm{~B} 10$, and $6 \mathrm{C} 6$ diverged from a common germline $\mathrm{Ab}$. While $\mathrm{Ab} 6 \mathrm{C} 8$ is differentiated from the common germline precursor by only a single mutation, suggesting that it was isolated during an early stage of its evolution, Abs $8 \mathrm{~B} 10$ and $6 \mathrm{C} 8$ are differentiated by four and five mutations, respectively, which is more typical for Abs isolated via similar protocols. ${ }^{42-45}$ Regardless of their state of affinity maturation, the unique mutations in each $\mathrm{Ab}$ reveal that multiple pathways exist for the evolution of MPTS recognition, and the fact that each evolved from a common germline precursor allows for the differences between the Abs to be ascribed to their different pathways of evolution. Our analysis revealed that while each pathway optimized MPTS recognition, they did so in different ways, and as a result, each $\mathrm{Ab}$ possesses a rather different overall profile of molecular recognition. Nonetheless, a similar level of elasticity is observed for each $\mathrm{Ab}-\mathrm{MPTS}$ complex, which is perhaps not unexpected because each has a similar disposition of charged protein side chains and water molecules, and elasticity is largely insensitive to structural details. ${ }^{46}$ Thus, the differences in molecular recognition appear to result from changes in $\mathrm{Ab}$ structure, anelasticity, and/or plasticity.

$\mathrm{Ab}$ 6C8 has the lowest affinity for MPTS but binds MPTS with the most favorable entropy, and of the three siblings, it recognizes the broadest range of targets. The complex with MPTS shows no plasticity and has the lowest level of anelasticity of the siblings. $\mathrm{Ab} 6 \mathrm{C} 8$ acquired only the $\mathrm{I}^{\mathrm{L}} 30 \mathrm{~N}$ mutation during its evolution, and the structure reveals that this mutation installs an $\mathrm{H}$-bond with the third sulfonate group of MPTS (Figure 7A). This intermolecular H-bond likely forces 
A

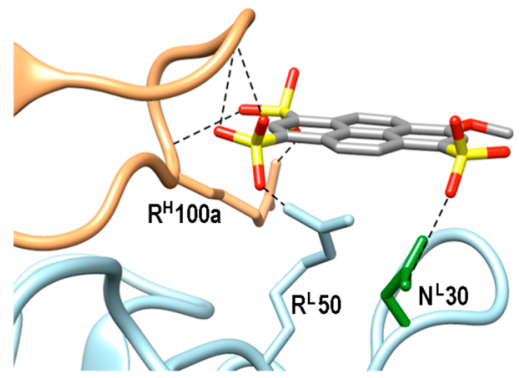

B
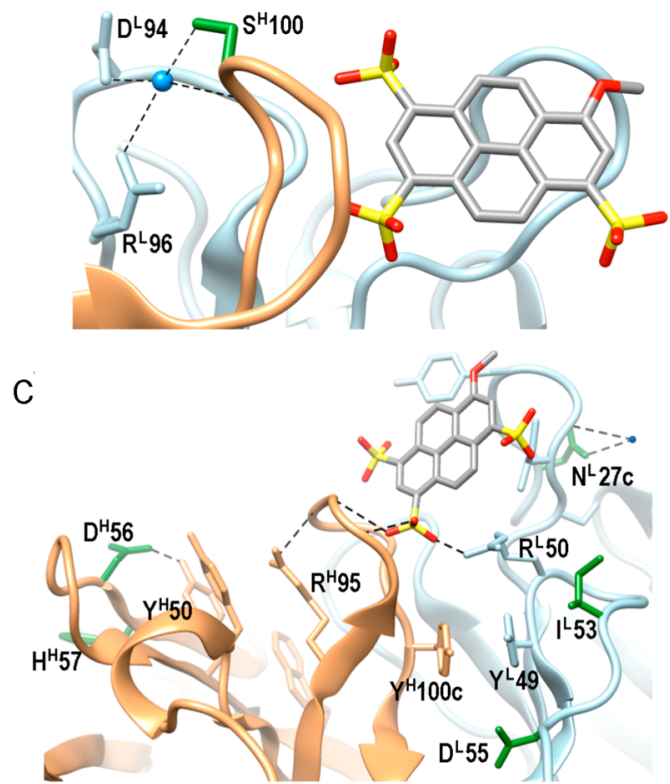

Figure 7. View of the MPTS binding site in the crystal structures of $A b$ 6C8 (A) and 8B10 (B) and the model structure of Ab 6C6 (C). The $\mathrm{V}_{\mathrm{H}}$ and $\mathrm{V}_{\mathrm{L}}$ are colored orange and light blue, respectively; side chains of somatic mutations are colored green, and waters in panels B and C are shown as blue spheres. Each $\mathrm{Ab}$ structure is viewed from an optimal perspective to visualize its unique set of somatic mutations.

desolvation of both the Ab and MPTS upon complex formation, which accounts for the more favorable binding entropy. In addition, relative to the already present $\mathrm{H}$-bonds, the new H-bond engages MPTS from the opposite side, which otherwise does not interact with the $\mathrm{Ab}$. Thus, combined, the H-bonds act as a pincer that clamps MPTS in place, which likely accounts for the low levels of anelasticity and plasticity observed with this complex. The rigidity of the complex is clearly dependent on the presence of MPTS and is not expected to be manifest in the free $\mathrm{Ab}$, where increased rigidity might be expected to restrict molecular recognition. In fact, the high polyspecificity of $\mathrm{Ab} 6 \mathrm{C} 8$ suggests that the free $\mathrm{Ab}$ is more flexible than its siblings, thereby allowing it to accommodate a large range of targets via an induced fit or conformational selection mechanism. This is consistent with $6 \mathrm{C} 8$ being only a single mutation removed from the presumably flexible and polyspecific germline $\mathrm{Ab}$. Along with its modest affinity for MPTS, this suggests that $\mathrm{Ab} 6 \mathrm{C} 8$ was isolated at an early stage of affinity maturation and that, if affinity matured further, would have acquired more mutations that rigidified its binding site and increased its affinity and specificity for MPTS.

$\mathrm{Ab} 8 \mathrm{~B} 10$ has an intermediate affinity for MPTS, but it is the most specific of the sibling Abs, showing little to no affinity for any of the randomly selected proteins. As with $6 \mathrm{C} 8$, the $8 \mathrm{~B} 10-$
MPTS complex shows no plasticity, but it does show significant anelasticity. $\mathrm{Ab} 8 \mathrm{~B} 10$ evolved via the acquisition of four somatic mutations, $\mathrm{N}^{\mathrm{L}} 76 \mathrm{I}, \mathrm{A}^{\mathrm{H}} 16 \mathrm{~T}, \mathrm{D}^{\mathrm{H}} 85 \mathrm{E}$, and $\mathrm{I}^{\mathrm{H}} 100 \mathrm{~S}$, none of which directly contact MPTS. The residues at positions ${ }^{\mathrm{L}} 76$ and ${ }^{\mathrm{H}} 85$ are solvent-exposed, and their mutation does not appear to introduce any structural changes relative to the other Abs. However, $\mathrm{A}^{\mathrm{H}} 16 \mathrm{~T}$ introduces a side-chain H-bond with the backbone carbonyl of Lys ${ }^{\mathrm{H}} 13$ that appears to stabilize a $\beta$-turn involving residues ${ }^{\mathrm{H}} 13-{ }^{\mathrm{H}} 16$. Residue ${ }^{\mathrm{H}} 100$ is located in a Glyrich sequence of $\mathrm{V}_{\mathrm{H}} \mathrm{CDR} 3$ and is flanked by residues that form H-bonds with the two proximal sulfonates of MPTS. The $\mathrm{I}^{\mathrm{H}} 100 \mathrm{~S}$ mutation of $\mathrm{Ab} 8 \mathrm{~B} 10$ leads to the introduction of an ordered water molecule that is not present in the structure of the other Abs and that forms $\mathrm{H}$-bonds that link the side chain and carbonyl backbone of $S^{\mathrm{H}} 100$ with the side chains of $\operatorname{Arg}^{\mathrm{L}} 96$ and Asp ${ }^{\mathrm{L}} 94$ (Figure 7B). While likely helping to order the combining site, the water-mediated $\mathrm{H}$-bonds are expected to permit significant small scale motion, which may contribute to the anelasticity of the 8B10-MPTS complex. Upon comparison of the $6 \mathrm{C} 8$ and $8 \mathrm{~B} 10$ complexes, it is instructive to note that the dye coumarin 153 shows a similar increase in the time scale of diffusive motion upon a 4-fold increase in solvent viscosity (from 4.2 ps in $\mathrm{CHCl}_{3}$ to $10.7 \mathrm{ps}$ in $\mathrm{DMSO}$ ), ${ }^{47}$ suggesting that the differences in anelasticity of the two complexes are functionally significant. Nonetheless, the watermediated interactions introduced are relatively directional, and any larger scale motions would require their rupture, consistent with the absence of plasticity of the complex. Unlike for $\mathrm{Ab}$ $6 \mathrm{C} 8$, the dynamics observed for the $8 \mathrm{~B} 10$ complex are likely to be manifest in the free $A b$, as none of the introduced mutations involve direct interactions with the Ag. Thus, the absence of plasticity likely explains the relatively high specificity of $\mathrm{Ab}$ $8 \mathrm{~B} 10$ and suggests that this $\mathrm{Ab}$ recognizes MPTS with more of a lock-and-key-like mechanism.

$\mathrm{Ab}$ 6C6 has the highest affinity for MPTS, and it shows a relatively high level of polyspecificity compared to $\mathrm{Ab} 8 \mathrm{~B} 10$ (although less so than Ab 6C8). Like the 8B10-MPTS complex, the 6C6-MPTS complex shows higher levels of anelasticity than the 6C8-MPTS complex, but the 6C6-MPTS complex is also the only complex to show a significant level of plasticity. $\mathrm{Ab}$ 6C6 evolved via the acquisition of five somatic mutations, $\mathrm{D}^{\mathrm{L}} 27 \mathrm{cN}, \mathrm{N}^{\mathrm{L}} 53 \mathrm{I}, \mathrm{E}^{\mathrm{L}} 55 \mathrm{D}, \mathrm{G}^{\mathrm{H}} 56 \mathrm{D}$, and $\mathrm{N}^{\mathrm{H}} 57 \mathrm{H}$, all of which are distal to the Ag (Figure 7C). The side chain of the residue at position ${ }^{\mathrm{H}} 57$ is solvent-exposed and unlikely to contribute to Ag binding. The $\mathrm{D}^{\mathrm{L}} 27 \mathrm{cN}$ mutation appears to disrupt an $\mathrm{H}$ bond with the backbone $\mathrm{N}$ of $\operatorname{Arg}^{\mathrm{L}} 68$, possibly stabilizing a water-mediated $\mathrm{H}$-bond to $\mathrm{Gly}^{\mathrm{L}} 29$, whose flanking residues pack against the Ag. The $\mathrm{N}^{\mathrm{L}} 53 \mathrm{I}$ somatic mutation increases packing interactions with $\mathrm{Tyr}^{\mathrm{L}} 49$, which in turn packs with the side chains of $\operatorname{Arg}^{\mathrm{L}} 50$ and $\operatorname{Tyr}^{\mathrm{H}} 100 \mathrm{c}$. $\operatorname{Arg}^{\mathrm{L}} 50 \mathrm{H}$-bonds with MPTS, and $\mathrm{Tyr}^{\mathrm{H}} 100 \mathrm{c}$ forms part of the floor of the binding site. $\mathrm{Tyr}^{\mathrm{H}} 100 \mathrm{c}$ also packs with the side chain of residue ${ }^{\mathrm{L}} 55$, and the $\mathrm{E}^{\mathrm{L}} 55 \mathrm{D}$ mutation appears to stabilize the unique orientation adopted by the $\mathrm{Tyr}^{\mathrm{H}} 100 \mathrm{c}$ side chain. The $\mathrm{G}^{\mathrm{H}} 56 \mathrm{D}$ mutation, located in $\mathrm{V}_{\mathrm{H}} \mathrm{CDR} 2$, introduces an $\mathrm{H}$-bond with the side chain of $\mathrm{Tyr}^{\mathrm{H}} 50$, which participates in a hydrophobic cluster that includes the side chain of $\operatorname{Arg}^{\mathrm{H}}$ 95. $\operatorname{Arg}^{\mathrm{H}}$ 95 forms an $\mathrm{H}$-bond with the backbone $\mathrm{O}$ of $\mathrm{Gly}^{\mathrm{H}} 99$, which forms part of the supporting structure behind the main walls of the Ag binding site. While all of these interactions may help to increase the affinity for MPTS, none of the somatic mutations directly contact the $\mathrm{Ag}$, and because most involve less directional packing interactions, both small and larger scale distortions 
should be possible, accounting for the observed anelasticity, which is similar to that of $\mathrm{Ab} 8 \mathrm{~B} 10$, and the observed plasticity, which is unique to this $\mathrm{Ab}$. To gauge whether the level of plasticity is likely to be biologically relevant, we note that $\Delta_{\text {inh }}$ for chromophores in small molecule solvents is invariably zero and can range up to only $\sim 500 \mathrm{~cm}^{-1}$ in acrylic glasses, which possess perhaps the largest possible range of static environments. ${ }^{48,49}$ Thus, the variation in static inhomogeneity of the $\mathrm{Ab}-\mathrm{MPTS}$ complexes is more than one-third the difference between a free solvent and a glass environment, which implies that the differences in plasticity that they reflect are biologically significant. As with $\mathrm{Ab} 8 \mathrm{~B} 10$, the dynamics observed for the 6C6 complex are likely to be manifest in the free $\mathrm{Ab}$, as none of the introduced mutations involve interactions with the Ag. Thus, the high level of plasticity likely underlies the broad polyspecificity observed with this $\mathrm{Ab}$, as it should facilitate the recognition of different targets with a conformational selection mechanism of molecular recognition.

The results demonstrate that a single progenitor $\mathrm{Ab}$ may be optimized by affinity maturation to better recognize MPTS via a variety of different pathways. With $\mathrm{Ab} 6 \mathrm{C} 8$, affinity maturation installed a single functional group that directly interacts with MPTS. While the affinity maturation of Abs $8 \mathrm{~B} 10$ and 6C6 involved the introduction of more mutations, the effects on structure were more subtle, and the mutations have larger effects on anelasticity and plasticity. Moreover, the fact that the less plastic $\mathrm{Ab}$ shows greater selectivity is consistent with the model in which a restriction in plasticity is a critical component of affinity maturation, as high specificity allows mature $\mathrm{Abs}$ to be produced at the high levels required to eradicate an infection without causing autoimmunity. ${ }^{26-28}$ Regardless, the data suggest that both structure and dynamics are affected by affinity maturation and possibly that both contribute to the optimization of molecular recognition. A more direct and quantitative analysis of the optimization requires cloning, expression, and characterization of the germline $A b$, which is currently limited by poor expression yields. While these studies employed a small molecule Ag, and small molecule and protein $\mathrm{Ag}$ often elicit different types of combining sites, it seems likely that the underlying mechanism(s) by which recognition is optimized may be similar. Moreover, a similar optimization of structure and dynamics likely contributes to the optimization of molecular recognition mediated by other proteins, and thus to the evolution of novel function, in general.

\section{ASSOCIATED CONTENT}

\section{S Supporting Information}

Supporting methods, tables, and figures; data analysis; nucleotide sequences of the $\mathrm{Ab}$ variable-chain genes; and supporting references. This material is available free of charge via the Internet at http://pubs.acs.org.

\section{AUTHOR INFORMATION}

\section{Corresponding Author}

*E-mail: floyd@scripps.edu. Telephone: (858) 784-7290. Fax: (858) 784-7472.

\section{Funding}

Funding was provided by the National Institute of Allergy and Infectious Diseases (AI079319). Crystallographic data were collected at APS GM/CA that has been funded in whole or in part with federal funding from the National Cancer Institute
(Y1-CO-1020) and the National Institute of General Medical Sciences (Y1-GM-1104). Use of the Advanced Photon Source was supported by the U.S. Department of Energy, Basic Energy Sciences, Office of Science, under Contract DE-AC0206CH11357.

\section{Notes}

The authors declare no competing financial interest.

\section{ACKNOWLEDGMENTS}

We thank Julie Vanhasy and Kendra Pivaroff-Ward for Fab preparation and purification.

\section{REFERENCES}

(1) Patten, P. A., Gray, N. S., Yang, P. L., Marks, C. B., Wedemayer, G. J., Boniface, J. J., Stevens, R. C., and Schultz, P. G. (1996) The immunological evolution of catalysis. Science 271, 1086-1091.

(2) Wedemayer, G. J., Patten, P. A., Wang, L. H., Schultz, P. G., and Stevens, R. C. (1997) Structural insights into the evolution of an antibody combining site. Science 276, 1665-1669.

(3) Ortlund, E. A., Bridgham, J. T., Redinbo, M. R., and Thornton, J. W. (2007) Crystal structure of an ancient protein: Evolution by conformational epistasis. Science 317, 1544-1548.

(4) Koshland, D. E., Jr. (1958) Application of a theory of enzyme specificity to protein synthesis. Proc. Natl. Acad. Sci. U.S.A. 44, 98-104.

(5) Foote, J., and Milstein, C. (1994) Conformational isomerism and the diversity of antibodies. Proc. Natl. Acad. Sci. U.S.A. 91, 1037010374.

(6) Ma, B., Shatsky, M., Wolfson, H. J., and Nussinov, R. (2002) Multiple diverse ligands binding at a single protein site: A matter of pre-existing populations. Protein Sci. 11, 184-197.

(7) Bosshard, H. R. (2001) Molecular recognition by induced fit: How fit is the concept? News Physiol. Sci. 16, 171-173.

(8) Fischer, E. (1894) Einfluss der Configuration auf die Wirkung der Enzyme. Ber. Dtsch. Chem. Ges. 27, 2985-2993.

(9) Billington, E. W., and Tate, A. (1981) The Physics of Deformation and Flow, pp 1-626, McGraw-Hill International, New York.

(10) Marcinkowski, M. J. (1979) Unified Theory of the Mechanical Behavior of Matter, pp 1-260, Wiley, New York.

(11) Soutas-Little, R. W. (1973) Elasticity, pp 1-448, Dover Publications, New York.

(12) Kachanov, L. M. (1971) Fundamentals of the Theory of Plasticity, pp 1-510, Dover Publications, New York.

(13) David, L., Quinson, R., Gauthier, C., and Perez, J. (1997) The role of anelasticity in high stress mechanical response and physical properties of glassy polymers. Polym. Eng. Sci. 37, 1633-1640.

(14) Oleynik, E. (1989) Plastic deformation and mobility in glassy polymers. Prog. Colloid Polym. Sci. 80, 140-150.

(15) Northrup, S. H., and Erickson, H. P. (1992) Kinetics of proteinprotein association explained by Brownian dynamics computer simulation. Proc. Natl. Acad. Sci. U.S.A. 89, 3338-3342.

(16) Camacho, C. J., Kimura, S. R., DeLisi, C., and Vajda, S. (2000) Kinetics of desolvation-mediated protein-protein binding. Biophys. J. $78,1094-1105$.

(17) Cho, M., Yu, J.-Y., Joo, T., Nagasawa, Y., Passino, S. A., and Fleming, G. R. (1996) The integrated photon echo and solvation dynamics. J. Phys. Chem. 100, 11944-11953.

(18) de Boeij, W. P., Pshenichnikov, M. S., and Wiersma, D. A. (1996) System-bath correlation function probed by conventional and time-grated stimulated photon echo. J. Phys. Chem. 100, 11806-11823.

(19) Fleming, G. R., and Cho, M. H. (1996) Chromophore-solvent dynamics. Annu. Rev. Phys. Chem. 47, 109-134.

(20) Mukamel, S. (1995) Principles of Nonlinear Optical Spectroscopy, Oxford University Press, New York.

(21) Landsteiner, K. (1962) The Specificity of Serological Reactions, Dover, Mineola, NY.

(22) Tonegawa, S. (1983) Somatic generation of antibody diversity. Nature 302, 575-581. 
(23) Berek, C., and Milstein, C. (1987) Mutation drift and repertoire shift in the maturation of the immune response. Immunol. Rev. 96, 2341.

(24) Berek, C., and Milstein, C. (1988) The dynamic nature of the antibody repertoire. Immunol. Rev. 105, 5-26.

(25) Steele, E. J., Ed. (1990) Somatic Hypermutation in V-Regions, CRC Press, Boca Raton, FL.

(26) Jimenez, R., Case, D. A., and Romesberg, F. E. (2002) Flexibility of an antibody binding site measured with photon echo spectroscopy. J. Phys. Chem. B 106, 1090-1103.

(27) Jimenez, R., Salazar, G., Yin, J., Joo, T., and Romesberg, F. E. (2004) Protein dynamics and the immunological evolution of molecular recognition. Proc. Natl. Acad. Sci. U.S.A. 101, 3803-3808.

(28) Zimmermann, J., Oakman, E. L., Thorpe, I. F., Shi, X. H., Abbyad, P., Brooks, C. L., Boxer, S. G., and Romesberg, F. E. (2006) Antibody evolution constrains conformational heterogeneity by tailoring protein dynamics. Proc. Natl. Acad. Sci. U.S.A. 103, 1372213727.

(29) Thielges, M. C., Zimmermann, J., Yu, W., Oda, M., and Romesberg, F. E. (2008) Exploring the energy landscape of antibodyantigen complexes: Protein dynamics, flexibility, and molecular recognition. Biochemistry 47, 7237-7247.

(30) Adhikary, R., Yu, W., Oda, M., Zimmermann, J., and Romesberg, F. E. (2012) Protein dynamics and the diversity of an antibody response. J. Biol. Chem. 287, 27139-27147.

(31) Harlow, E., and Lane, D. (1988) Antibodies: A Laboratory Manual, pp 371-396, Cold Spring Harbor Laboratory Press, Plainview, NY.

(32) Sastry, L., Alting-Mees, M., Huse, W. D., Short, J. M., Sorge, J. A., Hay, B. N., Janda, K. D., Benkovic, S. J., and Lerner, R. A. (1989) Cloning of the immunological repertoire in Escherichia coli for generation of monoclonal catalytic antibodies: Construction of a heavy chain variable region-specific cDNA library. Proc. Natl. Acad. Sci. U.S.A. $86,5728-5732$.

(33) Ulrich, H. D., Patten, P. A., Yang, P. L., Romesberg, F. E., and Schultz, P. G. (1995) Expression studies of catalytic antibodies. Proc. Natl. Acad. Sci. U.S.A. 92, 11907-11911.

(34) McCoy, A. J., Grosse-Kunstleve, R. W., Adams, P. D., Winn, M. D., Storoni, L. C., and Read, R. J. (2007) Phaser crystallographic software. J. Appl. Crystallogr. 40, 658-674.

(35) Murshudov, G. N., Vagin, A. A., and Dodson, E. J. (1997) Refinement of macromolecular structures by the maximum-likelihood method. Acta Crystallogr. D53, 240-255.

(36) Emsley, P., Lohkamp, B., Scott, W. G., and Cowtan, K. (2010) Features and development of Coot. Acta Crystallogr. D66, 486-501.

(37) Cunningham, A. J. (1976) The Generation of Antibody Diversity: A New Look, pp 1-211, Academic Press, London.

(38) Alt, F. W., Blackwell, K., and Yancopoulos, G. D. (1987) Development of the primary antibody repertoire. Science 238, 10791087.

(39) Lefranc, M. P., Giudicelli, V., Ginestoux, C., Jabado-Michaloud, J., Folch, G., Bellahcene, F., Wu, Y., Gemrot, E., Brochet, X., Lane, J., Regnier, L., Ehrenmann, F., Lefranc, G., and Duroux, P. (2009) IMGT, the international ImMunoGeneTics information system. Nucleic Acids Res. 37, D1006-D1012.

(40) Kabat, E. A. (1983) Sequences of Proteins of Immunological Interest, pp 1-323, National Institutes of Health, U.S. Department of Health and Human Services, Bethesda, MD.

(41) Connolly, M. L. (1993) The molecular surface package. J. Mol. Graphics 11, 139-141.

(42) McKean, D., Huppi, K., Bell, M., Staudt, L., Gerhard, W., and Weigert, M. (1984) Generation of antibody diversity in the immune response of $\mathrm{BALB} / \mathrm{c}$ mice to influenza virus hemagglutinin. Proc. Natl. Acad. Sci. U.S.A. 81, 3180-3184.

(43) Berek, C., and Milstein, C. (1988) The dynamic nature of the antibody repertoire. Immunol. Rev. 105, 5-26.

(44) Jacob, J., Przylepa, J., Miller, C., and Kelsoe, G. (1993) In situ studies of the primary immune response to (4-hydroxy-3-nitrophenyl)- acetyl. III. The kinetics of $\mathrm{V}$ region mutation and selection in germinal center B cells. J. Exp. Med. 178, 1293-1307.

(45) Lu, Y. F., and Cerny, J. (2002) Repertoire of antibody response in bone marrow and the memory response are differentially affected in aging mice. J. Immunol. 169, 4920-4927.

(46) Baker, S. P., Vinci, R. P., and Arias, T. (2002) Elastic and anelastic behavior of materials in small dimensions. MRS Bull. 27, 2629.

(47) Horng, M. L., Gardecki, J. A., Papazyan, A., and Maroncelli, M. (1995) Subpicosecond measurements of polar solvation dynamics: Coumarin 153 revisited. J. Phys. Chem. 99, 17311-17337.

(48) Passino, S. A., Nagasawa, Y., Joo, T., and Fleming, G. R. (1997) Three-pulse echo peak shift studies of polar solvation dynamics. J. Phys. Chem. A 101, 725-731.

(49) Nagasawa, Y., Passino, S. A., Joo, T., and Fleming, G. R. (1997) Temperature dependence of optical dephasing in an organic polymer glass (PMMA) from 300 to $30 \mathrm{~K}$. J. Chem. Phys. 106, 4840-4852.

(50) Kabat, E. A., and Wu, T. T. (1991) Identical V region amino acid sequences and segments of sequences in antibodies of different specificities. Relative contributions of VH and VL genes, minigenes, and complementarity-determining regions to binding of antibodycombining sites. J. Immunol. 147, 1709-1719. 